\title{
Enhanced Images Underwater with Clay Particles
}

\author{
Ameen A. Noor ${ }^{1}$, Ziad M. Abood $^{2}$ and Hussein Abed ${ }^{3}$ \\ ${ }^{1,2}$ College of Education/ Mustansiriyah University \\ ${ }^{2}$ College of Education/ Mustansiriyah University \\ ${ }^{3}$ Mustansiriyah University \\ Baghdad- Iraq
}

\begin{abstract}
Underwater images are subjected to a number of external influences that cause blurry of the image due to water density, refraction of light in the water and inaccuracy of colors due to factors such as small objects and clay particles, so many researches have been carried out in the image enhancement affected by dust and improving underwater images.

In this paper, underwater images were taken with two different mobile phones (iPhone 7 s plus and Galaxy10 +) and different dimensions with clay atoms, and enhancement images using the Contrast Limited Adaptive Histogram Equalization (CLAHE), and Fuzzy intensification operators (Fuzzy INT) algorithms. A number of quality measurements were measured for the image after enhanced. It showed that the images in the iPhone were of higher quality for the fuzzy algorithm. The comparison with the previous works also showed that the current work gave better results, then combining the enhanced of the underwater images with clay particles.
\end{abstract}

Keywords: CLAHE, Fuzzy intensification operators, Underwater Images.

\section{INTRODUCTION}

Modern research has specialized in processing digital images in the process of increasing the quality of the image to clear and interpret its features accurately, and to get a better appearance after the impact of this image. Noise and poor variance and customs and rituals taken in that image to be able to measure the quality of the image by many quality standards used to ensure of the importance of improving the techniques recommended in the presentation, as for our humble research of the process of taking pictures of the body of underwater, the effects will be more complex because of the weather under the water from the dispersion of light before reaching the camera and the reflection of light and absorption and to the average Spyware [1]. In addition to fog or dust that is between the camera and the body which leads to making the image is not clear to people where visibility is different from one person to another.

\section{PROPOSE MODEL}

The proposed method consists of many phases as shown in Figure 1. These steps include: Take pictures underwater with clay particles with two modern mobile phones and in more than one angle and different dimensions, then applying CLAHE and fuzzy algorithms, then applying some image quality standards.

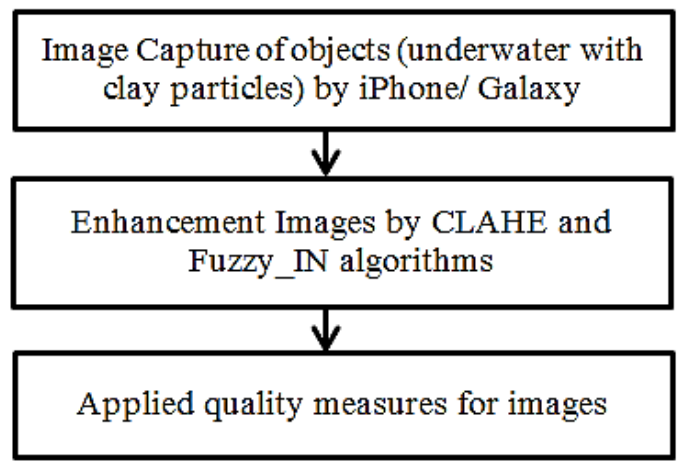

Figure 1. Block diagram of the Proposed Method 


\section{QUALITY MEASURES}

Many of quality measures were applied to the images, to measure the quality of optimization of the two methods (CLAHE and fuzzy). [5]

- MSE: is a quota of the value of an estimator-it is permanently non-negative, and values nearer to zero are well.

$$
\operatorname{MSE}=\frac{1}{n} \sum_{i=1}^{n}\left(y_{i}-\tilde{y}_{i}\right)^{2}
$$

- PSNR: process calculates the signal-to-noise ratio, where higher value is better; PSNR is generally conveyed in terms of the logarithmic decibel scale.

$$
P S N R=10 \cdot \log _{10}\left(\frac{M A X_{I}^{2}}{M S E}\right)
$$

- RMSE: The rectangular reason for Mean Square Error (MSE) involving the uniquely image $x$ (i, $j$ ) in addition the optimized image (reconstructed image) y $(i, j)$ because the lowermost RMSE value indicates that typically the image takes superior quality. RMSE is definite as follow:

$$
\text { RMSE }=\sqrt{\frac{1}{n} \sum_{j=1}^{n}\left(y_{j}-\hat{y}_{j}\right)^{2}}
$$

\section{FUZZY_IN ALGORITHM}

A fuzzy image is improved by setting the image intensity or the intensity of the color level in a mysterious plane using the organic function, then modifying these member functions to improve the contrast and assigning the dark plane to the image intensity or color intensity. The essence of improving the obscure image is the mid step where we adjust the membership values. Expert knowledge is considered to determine the best appropriate job for the operative and the operator adjusting the membership function. [6]

A technique used to control the accuracy of the color of the image being processed so that the image is split into channels (R, G, B) to compute the condensation factor, and the Tau factor is needed, which is the minimum operator value and the organic value of each channel between the 0 and 1, as follows: [7]

$$
\begin{aligned}
f_{\text {red }} & =\frac{[r-\min (r)]}{[\max (r)-\min (r)]} \\
f_{\text {grn }} & =\frac{[\mathrm{g}-\min (\mathrm{g})]}{[\max (\mathrm{g})-\min (\mathrm{g})]} \\
f_{b l k} & =\frac{[b-\min (b)]}{[\max (b)-\min (b)]}
\end{aligned}
$$

The value of the condensation factor is calculated using following formulas:

$$
\begin{array}{r}
K_{\text {red }}=2 *\left(f_{\text {red }}(\mathrm{x}, \mathrm{y})\right)^{2} \text { if } f_{\text {red }}(\mathrm{x}, \mathrm{y}) \leq \mathrm{Tred} \\
\text { Otherwise } 1-2 *\left(1-f_{\text {red }}(\mathrm{x}, \mathrm{y})\right)^{2} \\
K_{\text {grn }}=2 *\left(f_{g r n}(\mathrm{x}, \mathrm{y})\right)^{2} \text { if } f_{g r n}(\mathrm{x}, \mathrm{y}) \leq \mathrm{T}_{\text {grn }} \\
\text { Otherwise } 1-2 *\left(1-f_{\text {grn }}(\mathrm{x}, \mathrm{y})\right)^{2} \\
K_{b l k}=2 *\left(f_{b l k}(\mathrm{x}, \mathrm{y})\right)^{2} \text { if } f_{b l k}(\mathrm{x}, \mathrm{y}) \leq \mathrm{T}_{\text {blk }} \\
\text { Otherwise } 1-2 *\left(1-f_{b l k}(\mathrm{x}, \mathrm{y})\right)^{2}
\end{array}
$$

\section{CLAHE ALGORITHM}

Adaptive Histogram Equalization (AHE) calculates a graph from the local frame center in Specific pixels to specify the mapping for that pixel, providing a local improvement of contrast. Prevailing AHE, reducing the contrast limiting adaptive 
histogram equalization (CLAHE) [3,6] it has more flexibility in choosing a local mapping function. By selecting the graph snapshot level, the contrast-limited adaptive histogram equalization (CLAHE) prohibit the Excessive promotion by restricting stretching of alike gray levels in local image coverings. These ways advance the low-light image. [8]

Is a method of enhancing adaptive contrast depends on AHE. Where the graph of the contextual area of the pixel is calculated. The pixel intensity is therefore converted to a value inside the width range commensurate with the pixel strength rating CLAHE, has two main parameters: the mass size $(\mathrm{N})$ and the section limit (CL). These constraints are primarily used to controller image value, but are defined by users. CLAHE was originally established for medical imaging. CLAHE as claimed for advance better contrast in water improve aerial imagery [9]

This is attaining by reducing the contrast improvement of AHE. Contrast enlargement in the proximity of a given pixel value is given by the slope of the conversion function. This is proportionate to the slope of the adjacent cumulative distribution function (CDF), and thus to the graph value at this pixel value. CLAHE limits extension by cutting the graph with a Pre-defined value before calculating the CDF. This limits the inclination of CDF and thus of the shift function. The value at which the graph is clipped, which is called the stroke limit Figure 2, build on the standardization of the graph and therefore on the size of the vicinity area. Common values limit output amplification between 3 and 4 times average value of the graph. It's a good idea not to ignore the part of the graph that crosses the section boundary but redistribute it evenly across all the graph boxes. [8]

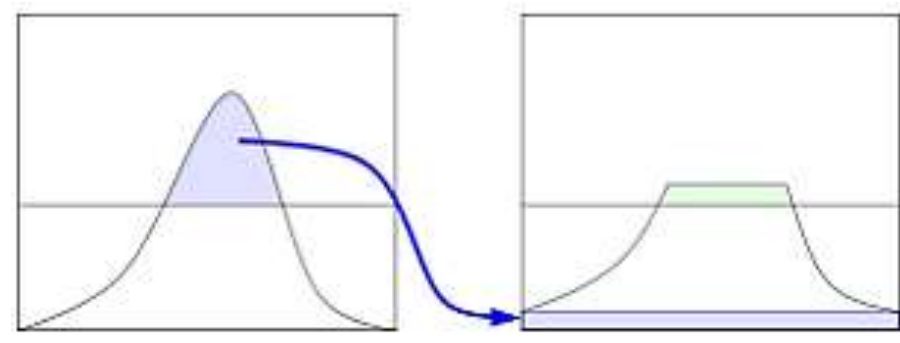

Figure 2 Redistributed Histogram

\section{RESULTS}

The results of images underwater ( iPhone7s plus + and Galaxy 10) before and after enhancement by HSV CLAHE and Fuzzy_INT methods, these results shown at Table 1 and Table 2.

Table 1. iPhone7s plus images underwater Database before and after enhancement (with different distance)

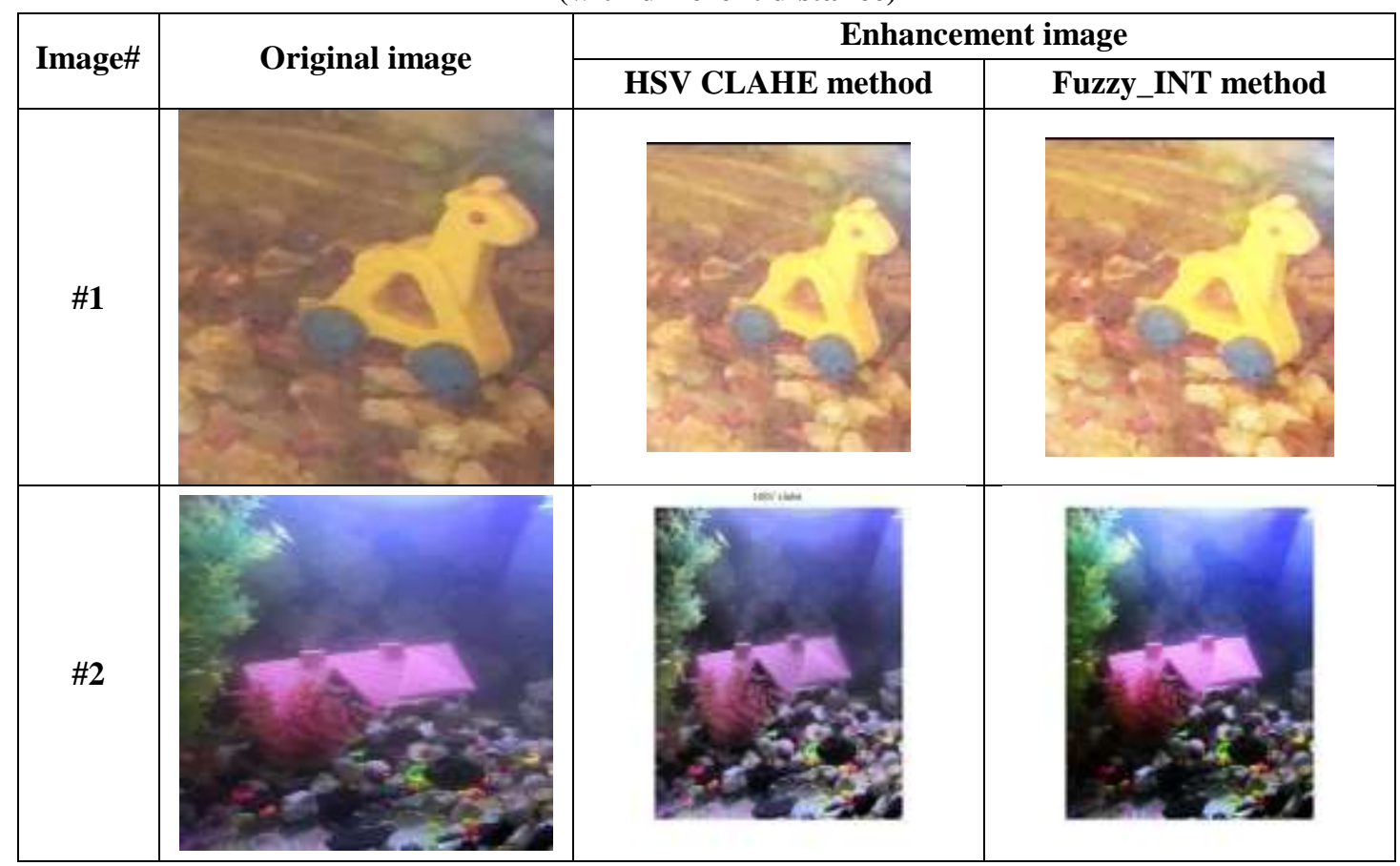


International Journal of Engineering Research And Advanced Technology, Vol.6, Issue 8, August-2020

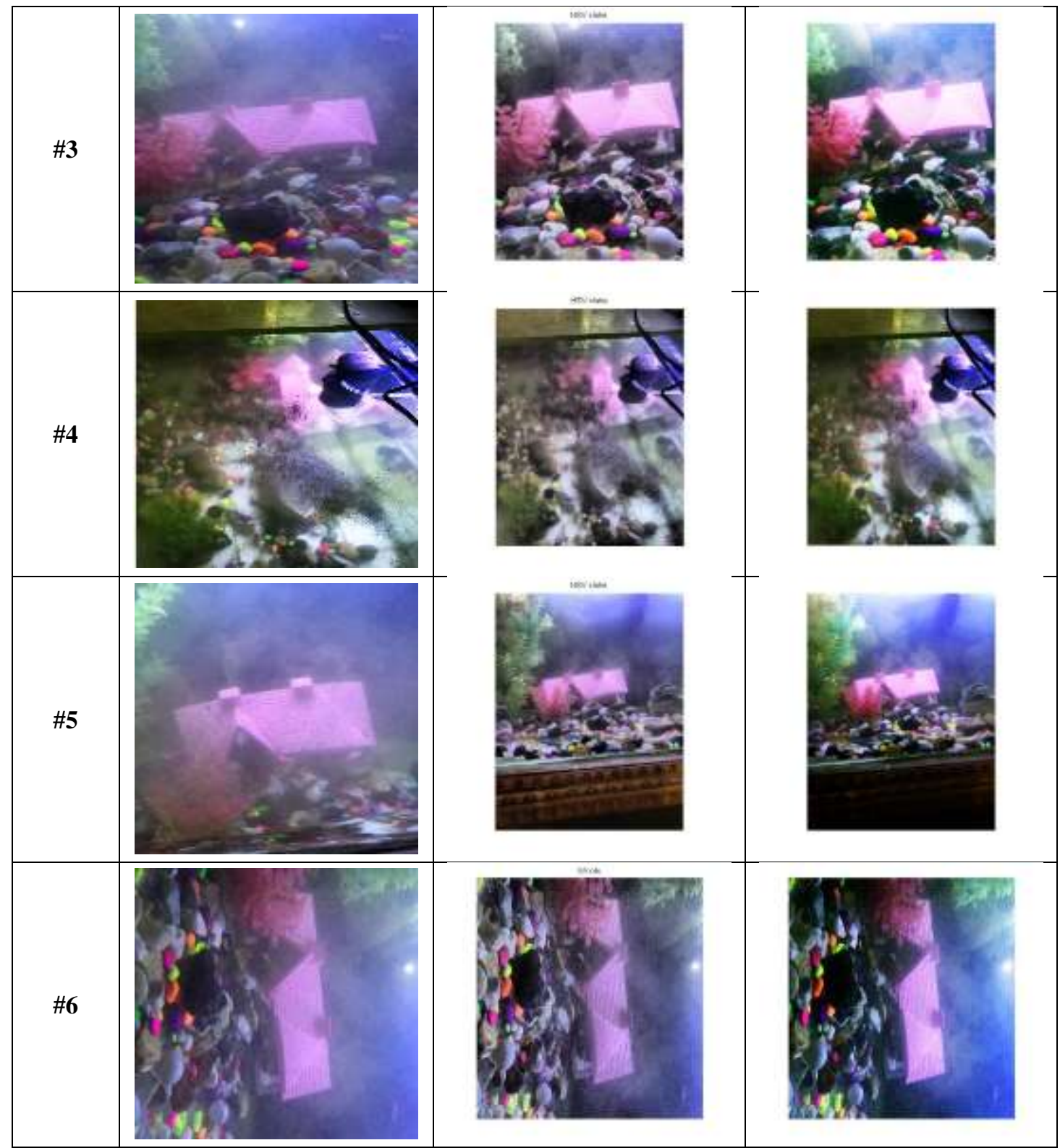


International Journal of Engineering Research And Advanced Technology, Vol.6, Issue 8, August-2020

Table 2. Galaxy 10 images underwater Database before and after enhancement (with different distance)

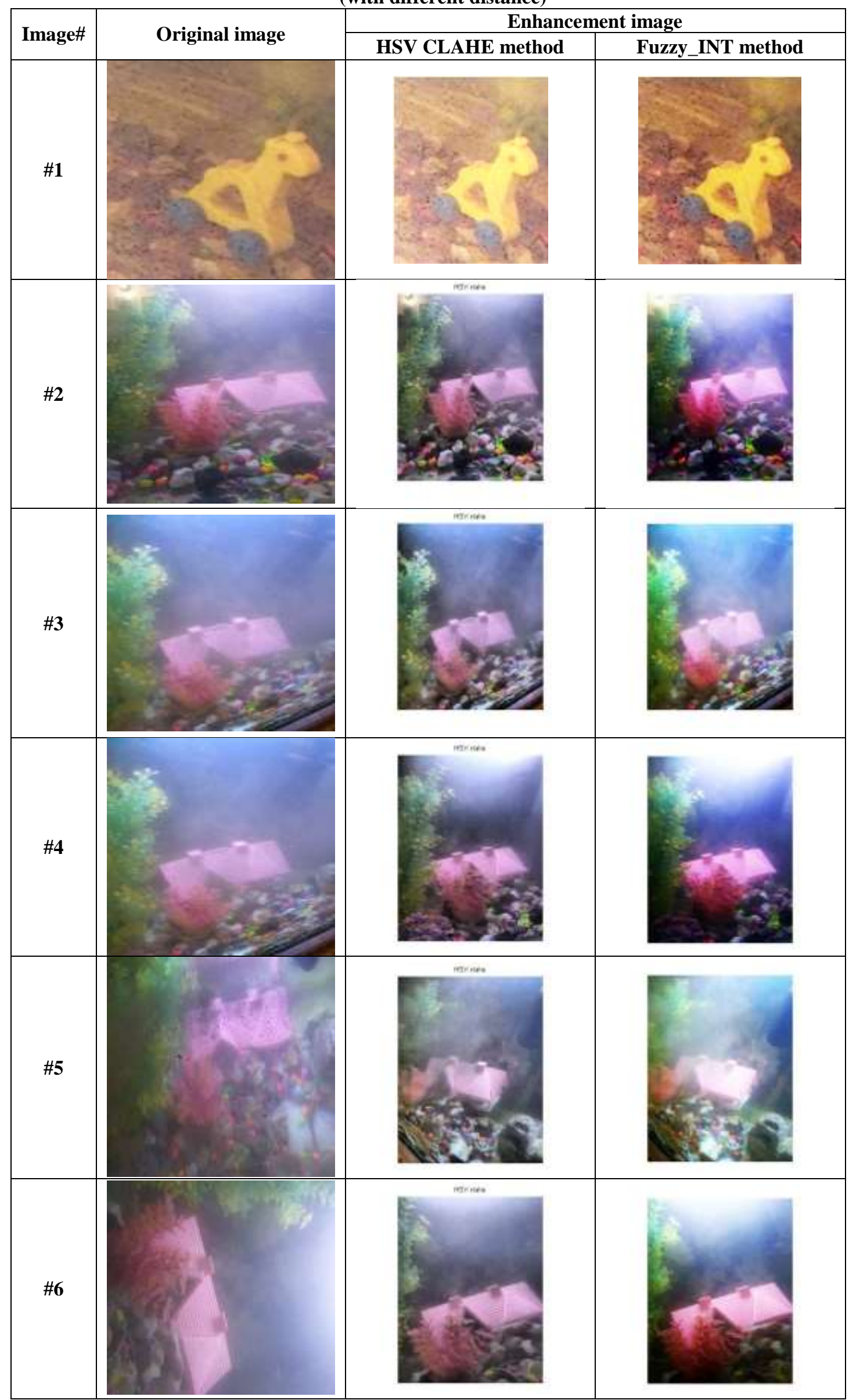


Image quality metric values for many of the medial image augmentation results shown in Table 3.

Table 3. Images quality measurements for Iphone7s plus and Galaxy 10

\begin{tabular}{|c|c|c|c|c|c|c|}
\hline Image\# & \multicolumn{3}{|c|}{ HSV CLAHE } & \multicolumn{3}{|c|}{ Fuzzy_INT } \\
\hline Iphone7S plus & RMSE & MSE & PSNR (dB) & RMSE & MSE & PSNR (dB) \\
\hline$\# 1$ & 107.70 & 0.1170 & 27.86 & 111.89 & 0.1170 & 29.37 \\
\hline$\# 2$ & 108.20 & 0.1160 & 27.46 & 100.25 & 0.1160 & 30.58 \\
\hline$\# 3$ & 139.30 & 0.1940 & 22.51 & 120.33 & 0.1940 & 21.23 \\
\hline$\# 4$ & 99.09 & 0.9819 & 28.09 & 99.09 & 0.9819 & 26.99 \\
\hline$\# 5$ & 117.95 & 0.1391 & 36.54 & 117.95 & 0.1391 & 39.74 \\
\hline \#6 & 106.46 & 0.1133 & 47.51 & 106.46 & 0.1133 & 48.25 \\
\hline Galaxy 10 & RMSE & MSE & PSNR (dB) & RMSE & MSE & PSNR (dB) \\
\hline$\# 1$ & 133.96 & 0.1794 & 31.93 & 127.43 & 0.1854 & 34.31 \\
\hline$\# 2$ & 137.83 & 0.1899 & 29.54 & 120.54 & 0.2145 & 30.31 \\
\hline$\# 3$ & 132.83 & 0.1764 & 27.27 & 139.74 & 0.1747 & 29.34 \\
\hline$\# 4$ & 144.00 & 0.2073 & 27.12 & 140.14 & 0.2157 & 28.47 \\
\hline$\# 5$ & 118.30 & 0.1399 & 36.47 & 115.45 & 0.1410 & 38.12 \\
\hline$\# 6$ & 145.12 & 0.2106 & 48.51 & 133.54 & 0.2197 & 46.10 \\
\hline
\end{tabular}

PSNR lacks the ability to evaluate image similarity across contrast-enhanced types. The PSNR value increases as the Improve brightness factor increases. Although the planned algorithm shows more detailed image information, this metric is similar in the case of CLAHE and Fuzzy_INT methods.

\section{CONCLUSIONS} conclusions:

After applied the HSV CLAHE and Fuzzy_INT on underwater images of present study, we can write the following

1. There is enhancement of underwater images after applying the two methods.

2. The Fuzzy_INT method gave better results than the HSV CLAHE.

3. The underwater images by Iphone7s plus were better than Galaxy 10A according to the image quality parameters.

\section{Acknowledgements}

The authors would like to thank Mustansiriyah University for supported (https://uomustansiriyah.edu.iq/) Baghdad- Iraq for its support in this work.

\section{REFERENCES}

[1] Ghada S. Karam, Ziad M. Abood, Rafal N. Saleh. Enhancement of underwater image using fuzzy histogram equalization, International Journal of Applied Information Systems, vol. 6, no. 8, 2013.

[2] Ramandeep Kaur, Dipen Saini, "Image Enhancement of Underwater Digital Images by Utilizing L*A*B* Color Space on Gradient and CLAHE based Smoothing", Communications on Applied Electronics (CAE). Foundation of Computer Science FCS, New York, USA vol. 4, No. 9, 2016.

[3] Garima Yadav, "Contrast limited adaptive histogram equalization based enhancement for real time video system", International Conference on Advances in Computing, Communications and Informatics (ICACCI). 2014.

[4] Zohair Al-Ameen, "Visibility Enhancement for Images Captured in Dusty Weather via Tuned Tri-threshold Fuzzy Intensification Operators", I. J. Intelligent Systems and Applications, vol. 8, pp. 10-17, 2016.

[5] Ramyashree N., Pathra P., Shruthi T. V., Jharna Majumdar, "Enhancement of Aerial and Medical Image using Multi resolution pyramid", Special Issue of IJCCT, vol. 1, Issue 2, 3, 4; International Conference - ACCTA. 2010.

[6] S. J. Preethi and K. Rajeswari, "Membership function modification for image enhancement using fuzzy logic," International Journal of Emerging Trends and Technology in Computer Science (IJETTCS), vol. 2, no. 2, 2013.

[7] Ziad M. Abood, Ghada S. Karam, Rafied E. Hluot, "Classification of Red Blood Cells Disease Using Fuzzy Logic Theory", Current Research in Computer Science and Information Technology (ICCIT), 2017.

[8] D. Bhadoriya, R. Gupta, M. Gupta. "Block Based Scheme using Tuned Tri-threshold Fuzzy Intensification Operators for Underwater Images”, International Journal of Computer Sciences and Engineering. vol. 7, Issue 2, 2019.

[9] D. P. Sharma, "Intensity Transformation using Contrast Limited Adaptive Histogram Equalization”, International Journal of Engineering Research, vol. 2, Issue 4, pp. 282-285, 2013. 